D.O.I.: $10.3895 / \mathrm{S} 1808-04482006000100004$

\title{
APLICAÇÃO DE SÉRIES TEMPORAIS NA SÉRIE TEOR DE UMIDADE DA AREIA DE FUNDIÇÃO DA INDÚSTRIA FUNDIMISA*
}

\section{APPLICATION OF TIME SERIES IN THE SÉRIE OF HUMIDITY TEXT IN THE INDÚSTRIA FUNDIMISA'S FOUNDRY SAND}

\author{
Suzana Russo'; Paulo M. M. Rodrigues ${ }^{2}$; Maria Emilia Camargo ${ }^{3}$ \\ ${ }^{1}$ Universidade Regional Integrada do Alto Uruguai e das Missões - URI Santo Ângelo - BRASIL - \\ jss@,urisan.tche.br \\ ${ }^{2}$ Faculdade de Economia - Universidade do Algarve - UALG - Faro - PORTUGAL - \\ prodrig@ualg.pt \\ ${ }^{3}$ Universidade de Caxias do Sul - UCS - Vacaria - BRASIL - kamargo@terra.com.br
}

\begin{abstract}
Resumo
Neste estudo verificam-se as alterações ocorridas nos moldes de fundição das peças produzidas pela indústria FUNDIMISA. Para tanto, é necessário encontrar um modelo que represente a realidade e faça previsões. Até o início dos anos 70, estudos envolvendo séries temporais procurava decompô-las suas componentes, após esta data foi criado uma abordagem alternativa, a metodologia Box e Jenkins, onde demonstra que as séries de tempo são integralmente geradas por um mecanismo aleatório. Para a modelagem ARIMA(p,d,q), deve-se determinar o número de sucessivas diferenciações para fazer com que a série original ( $Z$ ) se torne estacionária. Depois, determina-se a ordem da função e estima-se os parâmetros do modelo ARMA. Juntamente com responsáveis pelo processo produtivo, foi feita a determinação das variáveis referentes aos elementos envolvidos nos ensaios de areia para fundição, que são: teor de umidade, permeabilidade, resistência a compressão, compactabilidade e plasticidade da areia, coletadas no periodo de março/2004 até outubro/2004. Os dados usados são representativos do número diário analisado do teor de umidade da areia, medidos em \%. Observou-se que a série possui uma grande variabilidade, e fez-se a modelagem, onde o melhor modelo encontrado foi um AR(2), o critério de validação usado (MAPE) resultou 0,52\%, e o MSE foi 1,5.
\end{abstract}

Palavras-chave: Box e Jenkins, Séries temporais, Teor de umidade

* Estudo financiado pela CAPES e FAPERGS

\section{Introdução}

As recentes mudanças no mercado consumidor mundial têm exigido das empresas um grau máximo de racionalização, competitividade e modernização, tudo em busca de qualidade. Produtos com altos níveis de qualidade possuem maior durabilidade, o que, conseqüentemente, assegura uma 
boa imagem em frente aos clientes. Devido à esses fatores, este projeto de pesquisa objetiva aplicar a metodologia Box e Jenkins no processo de fabricação do molde de areia de fundição da Empresa FUNDIMISA - Fundição das Missões S.A., da cidade de Santo Ângelo - RS.

As análises de séries temporais no processo de fabricação de molde de areia são importantes para a melhor previsão das peças produzidas na empresa. Para análise dos dados dos ensaios de areia faz-se necessário que seja considerada a medição do teor de umidade executada no setor de qualidade.

\section{Objetivo}

Este trabalho tem como objetivo geral analisar através dos modelos de séries temporais a série teor de umidade da areia, para descrever o comportamento da série, encontrar a periodicidade nos dados e fazer previsões a curto prazo.

\section{Fundamentação teórica}

\subsection{Séries temporais}

A maior parte da teoria de séries temporais lida com séries estacionárias. Por isso, a série deve ser trabalhada previamente através de transformações. Se a série é aproximadamente estacionária, o processo gerador estacionário pode, então, ser adequadamente descrito pelos momentos de suas distribuições de probabilidade (BOX, JENKINS E REINSEL, 1994).

O estudo dos processos estacionários pode ser feito no domínio da freqüência ou no domínio do tempo. O estudo no domínio da freqüência dá papel de relevo aos conceitos de periodograma e de densidade espectral; o domínio no tempo atribui papel predominante às funções autocovariância e autocorrelação (BELTRÃO, 1991).

A autocorrelação é uma medida de dependência entre observações da mesma série separadas por um determinado intervalo chamado retardo. A função de autocorrelação (ACF) e a função de autocorrelação parcial (PACF) são a representação gráfica do coeficiente de autocorrelação e do coeficiente de autocorrelação parcial em função dos diversos retardos que podem ser atribuídos aos dados (BOX, JENKINS E REINSEL, 1994).

A tabela 1 apresenta um resumo dos padrões os quais permitem que se entenda melhor o

comportamento da dependência estatística entre os dados e, posteriormente, será útil para a determinação da ordem $p$ e $q$ estacionárias. 


\begin{tabular}{|c|c|c|}
\hline Modelo & $\mathrm{ACF}$ & PACF \\
\hline $\mathrm{AR}(\mathrm{p})$ & $\begin{array}{l}\text { Decaimento exponencial e/ou } \\
\text { senoidal amortecida para zero }\end{array}$ & Truncada no $\operatorname{lag} p$ \\
\hline $\operatorname{Ma}(q)$ & Truncada no $\operatorname{lag} q$ & $\begin{array}{l}\text { Decaimento exponencial ou senoidal } \\
\text { para zero }\end{array}$ \\
\hline $\operatorname{ARMA}(p . q)$ & $\begin{array}{l}\text { Decaimento exponencial ou senoidal } \\
\text { para zero depois do } \operatorname{lag} q\end{array}$ & $\begin{array}{l}\text { Decaimento exponencial ou senoidal } \\
\text { para zero depois do } \operatorname{lag} p\end{array}$ \\
\hline
\end{tabular}

O modelo básico de um processo estocástico é a sequencia de ruídos brancos $a_{t}$, onde $a_{t}$ é a variável aleatória independente com média zero e variância constante. Quando um processo não é um ruído branco, ele exibe uma dependência nos valores passados e tende a ser autocorrelacionados. A metodologia Box e Jenkins (Box; Jenkins, 1976) para séries temporais discretas serve para modelar esse comportamento autocorrelatado, e é baseada na idéia de que uma série temporal $Z$ no tempo $t=1,2,3, . . \mathrm{n}$, onde sucessivas observações são correlatadas, pode ser transformada em séries descorrelatadas de choques, ou de ruídos brancos, $a_{t}$.

Em muitas séries temporais há uma dependência (correlação) entre as variáveis, esta característica é verificada através dos modelos ARIMA (Autorregressivos-Integrados-MédiasMóveis). Estes modelos também seguem uma sequencia sistemática em cada estágio (identificação, estimação e diagnóstico) da modelagem (ZHANG, 2003).

\section{Resumo dos modelos:}

Modelos $\boldsymbol{A R}(\boldsymbol{p})$ : A classe dos modelos puramente autoregressivos é definido por:

$$
z_{t}=\frac{a_{t}}{\phi_{p}(B)}
$$

onde $f(B)$ tem $p$ coeficientes. O modelo $A R(p)$ pressupõe que seja o resultado da soma ponderada de seus $\mathrm{p}$ valores passados além do ruído branco ${ }_{t} a$. A condição de estacionariedade do $A R(p)$ estabelece que todas as $p$ raízes da equação característica caem fora do círculo unitário.

Modelos $\boldsymbol{M A}(\boldsymbol{q})$ : A classe dos modelos puramente médias móveis é definido por ${ }_{{ }_{q} t} Z=q(B) . a$, onde $q(B)$ tem $q$ coeficientes. Os modelos $M A(q)$ resultam da combinação linear dos choques aleatórios ocorridos no período corrente e nos períodos passados. A condição de invertibilidade requer que todas as raízes da equação característica caiam fora do círculo unitário.

Modelos $\boldsymbol{A R M A}(\boldsymbol{p}, \boldsymbol{q})$ : A classe dos modelos autoregressivos-médias-móveis é do tipo

$$
z_{t}=\frac{\theta_{q}(B) a_{t}}{\phi_{p}(B)}
$$

onde $f(B)$ tem $p$ coeficientes e $q(B)$ tem $q$ coeficientes. Com a combinação dos modelos $A R(p)$ e $M A(q)$, espera-se que os modelos $A R M A(p, q)$ sejam modelos extremamente parcimoniosos, usando 
poucos coeficientes para explicar a mesma sequencia. Do ponto de vista de ajuste, isso é muito importante, pois é possível ajustar mais rapidamente. A condição de estacionariedade e de invertibilidade de um $A R M A(p, q)$ requerem que todas as $p$ raízes de $f(B)=0$ e todas as $q$ raízes de $q(B)=0$ caiam fora do círculo unitário.

Modelos $\operatorname{ARIMA}(\boldsymbol{p}, \boldsymbol{d}, \boldsymbol{q})$ : A classe dos modelos-autoregressivos-integrados médias móveis é definido pela equação

$$
Z_{t}=\frac{\theta_{q}(B) a_{t}}{\phi_{p}(B)(1-B)^{d}}
$$

para um integrador positivo $d$. Após feita a diferenciação da série em d vezes necessárias para torná-la estacionária, o modelo $\operatorname{ARIMA}(p, d, q)$ pode ser ajustado através do modelo $A R M A(p, q)$ citado acima.

\section{Procedimentos metodológicos}

A metodologia utilizada foi a pesquisa teórico-empírica, usando-se as análises descritivas, exploratórias e explicativas. A pesquisa constou de um embasamento bibliográfico para levantar noções teóricas a respeito da metodologia estatística proposta.

O fluxograma da figura 1, apresenta o roteiro metodológico proposto a ser empregado na análise dos dados da Indústria FUNDIMISA, sendo um procedimento referencial para outras análises em outras empresas. Para o desenvolvimento do fluxograma esboçado na figura 1 , foi feito uma revisão da literatura de séries temporais.

As ferramentas auxiliares utilizadas, para a análise dos dados, foram os pacotes computacionais Statistica e o Excel. Utilizou-se o Excel para a análise exploratória dos dados e o pacote computacional Statistica para a modelagem Box e Jenkins.

Com os resultados obtidos neste trabalho, pretende-se apresentar um conjunto de diretrizes para a análise das outras variáveis proposta no projeto.

Uma das desvantagens/limitação dos modelos Box e Jenkins é a necessidade de uma grande quantidade de observações. Segundo Wei (1990) o número mínimo para um bom modelo deve ser 50 elementos (ONG et al, 2005). 
Figura 1 - Roteiro metodológico

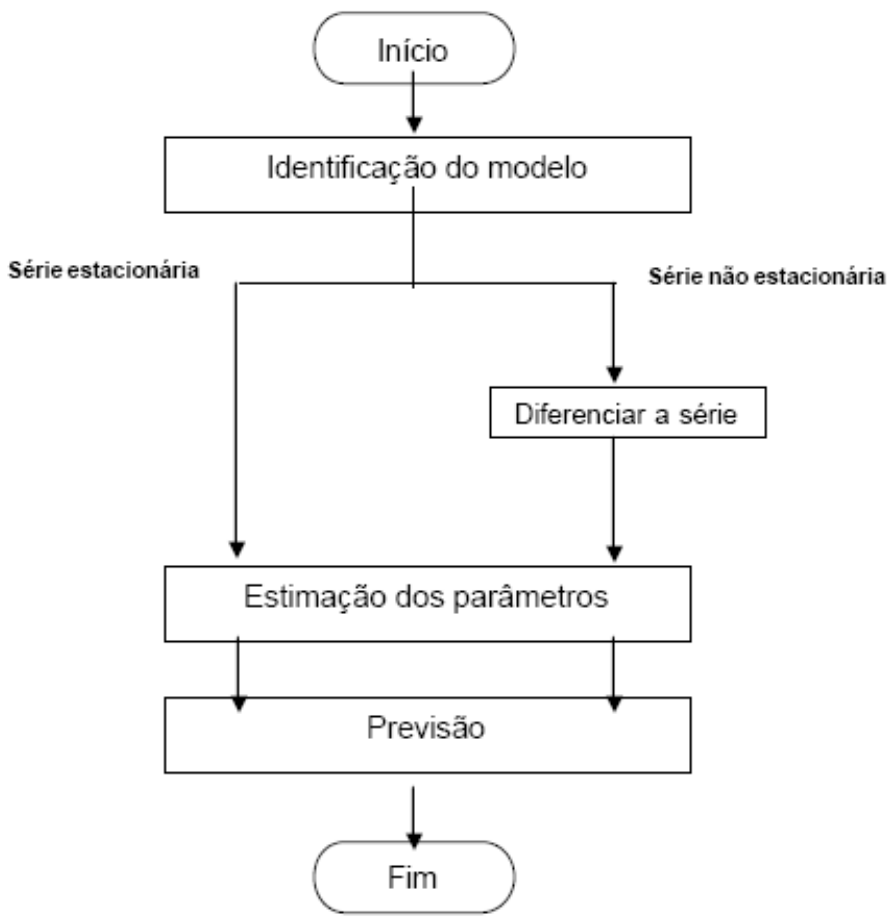

\section{Resultados e discussão}

No contato mantido com a equipe responsável pelo processo produtivo da indústria FUNDIMISA, foi feita a determinação das variáveis referentes aos elementos envolvidos na fundição, que são: teor de umidade, permeabilidade, resistência a compressão, compactabilidade e plasticidade da areia.

Essas variáveis formam as séries dos ensaios de areia base para fundição que foram coletadas no período disponibilizado pela empresa, de março/2004 até outubro/2004 (dados diários).

A análise de séries temporais do processo de fabricação de molde de areia é importante para a melhor qualidade das peças produzidas na empresa, sendo necessário encontrar um modelo que represente a simulação da realidade para se fazer previsões. Para análise dos dados dos ensaios de areia fez-se necessário considerar a medição do teor de umidade da areia, no referido período.

Os dados usados neste estudo são 195 valores representativos do número diário da plasticidade no período de março de 2004 a dezembro de 2004. A figura 2 mostra-se a série analisada, onde se observa que possui uma grande variabilidade. 
Figura 2 - Série teor da umidade da areia

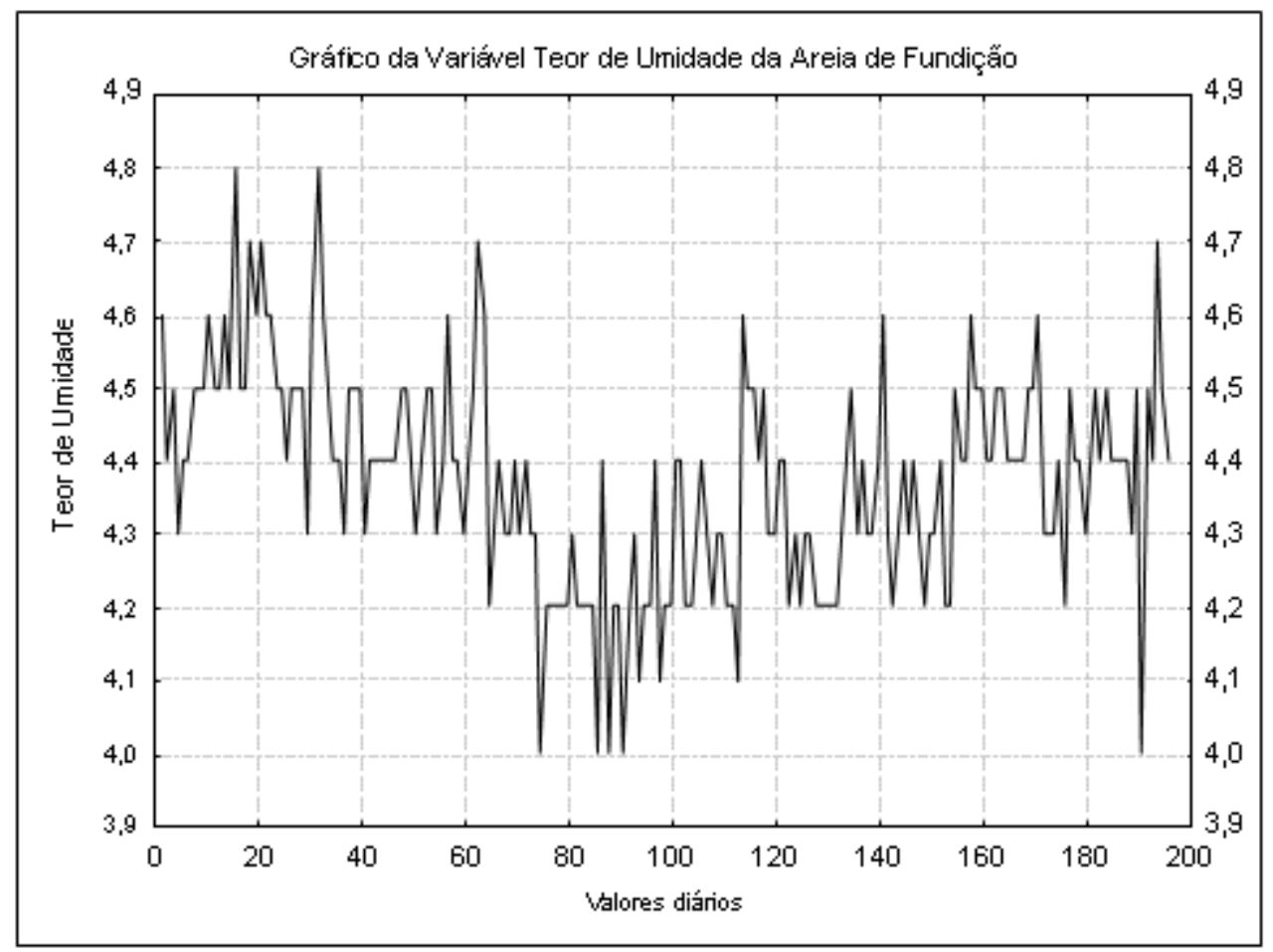

A tabela 2 mostra o resumo estatístico da série, onde se pode notar que a média é igual a $4,4 \%$, e o desvio padrão é 0,01 . Como a média, a mediana e a moda possuem o mesmo valor $(4,4 \%)$ a série é dita simétrica.

Tabela 2 - Resumo Estatístico

\begin{tabular}{lc}
\hline \multicolumn{1}{c}{ Medidas } & Medidas \\
\hline Média & $4,4 \%$ \\
Erro padrão & 0,01 \\
Mediana & $4,4 \%$ \\
Moda & $4,4 \%$ \\
Desvio padrão & 0,15 \\
Variância amostral & 0,02 \\
Contagem & 195 \\
Maior & $4,8 \%$ \\
Menor & $4,0 \%$ \\
\hline
\end{tabular}

\section{Identificação do modelo}

Verificou-se a autocorrelação dos dados através da função de autocorrelação e função de autocorrelação parcial representado nas figuras 3 e 4 respectivamente. 
Figura 3 - Função de autocorrelação para os dados observados

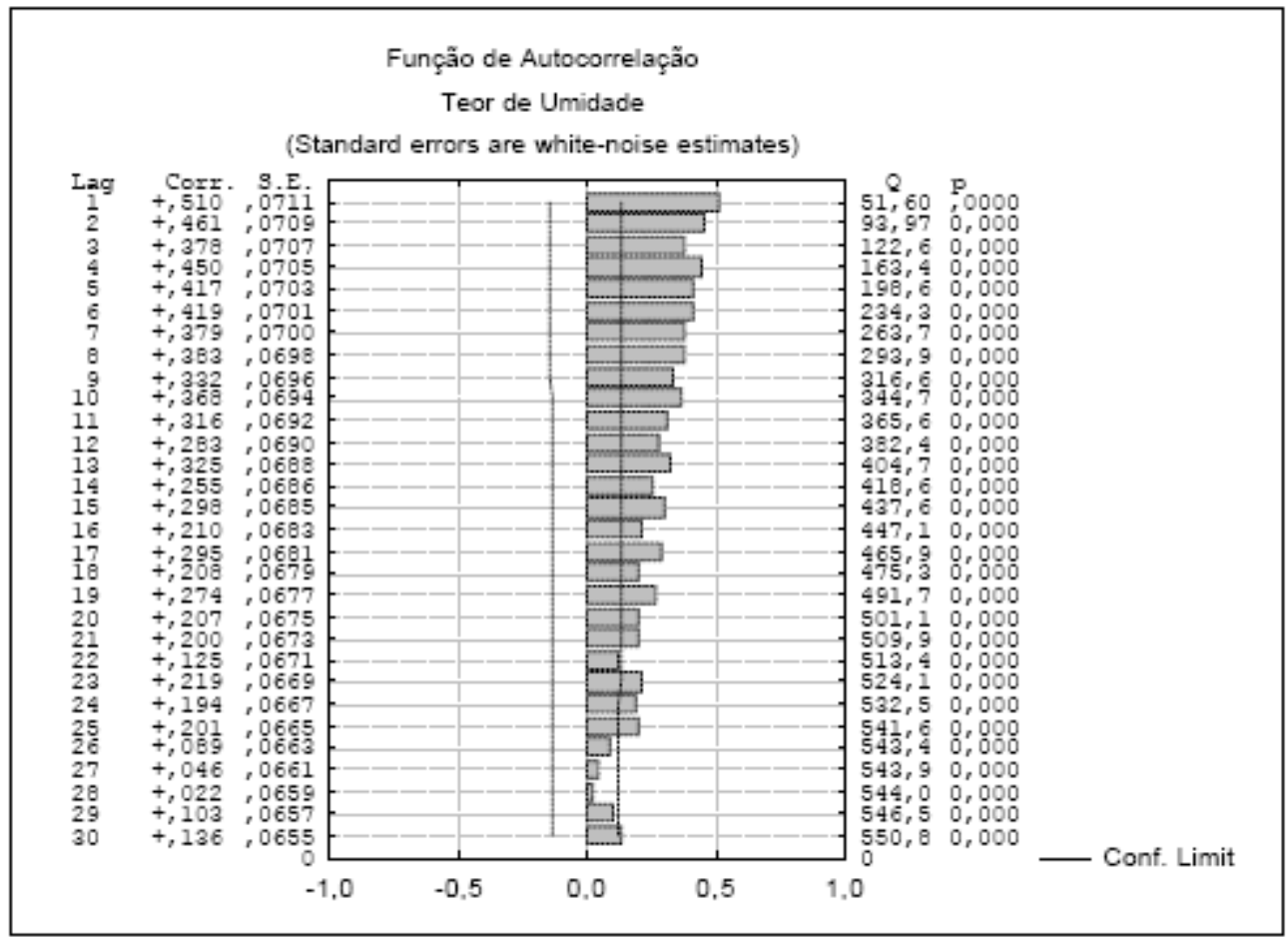

Figura 4 - Função de autocorrelação parcial para os dados observados

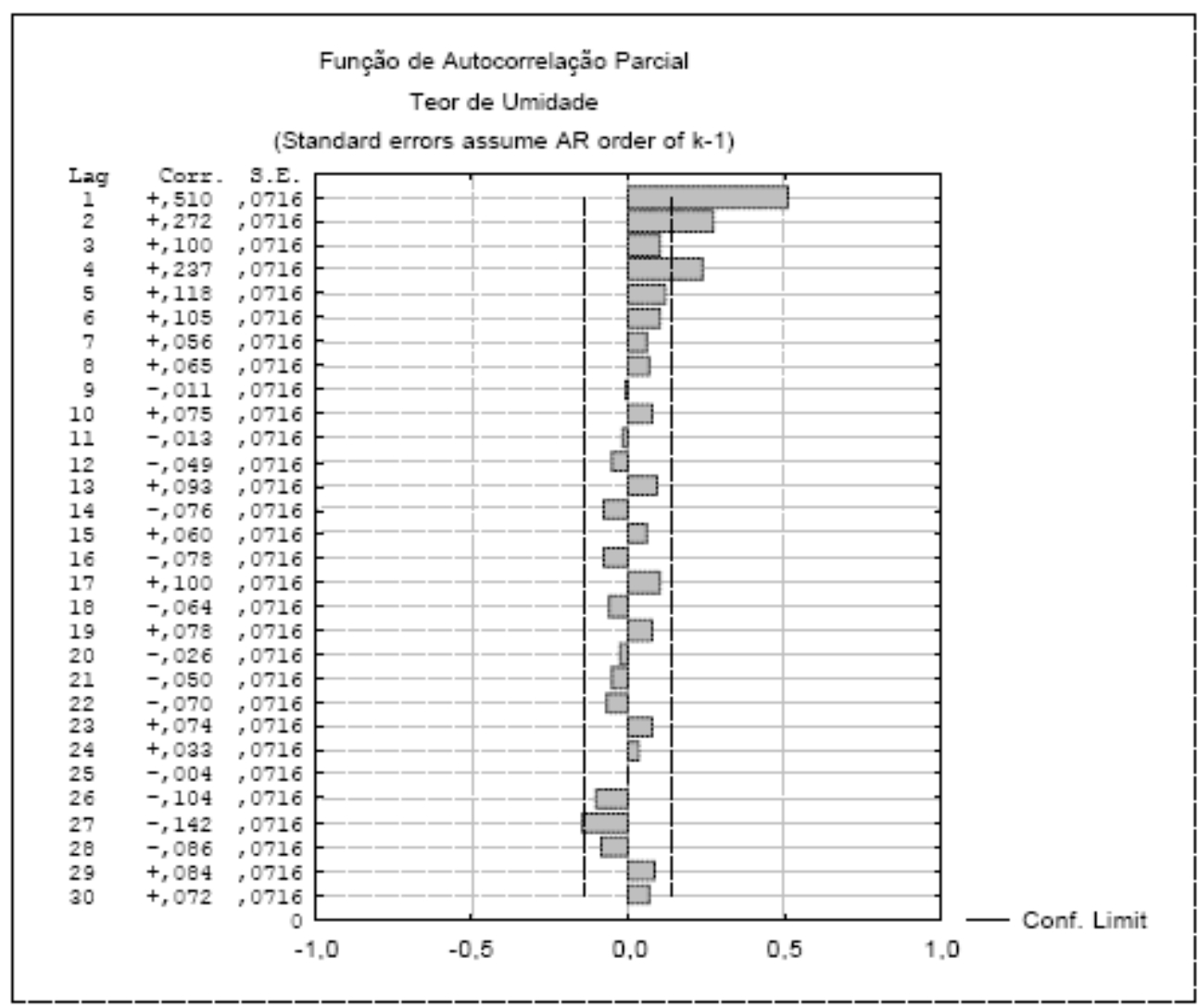

Através dos gráficos da função de autocorrelação exposto na figura 3, vê-se que os dados são autocorrelacionados, pois temos vários "lags" fora dos limites de controle e o gráfico da função de autocorrelação parcial exposto na figura 4, também mostra "lags" fora dos limites de controle, confirmando a autocorrelação da série. 


\section{Estimação dos parâmetros}

Modelou-se os dados através da metodologia de Box e Jenkins o que assegurou a independência das observações. Foi analisado a estacionariedade da série, verificando que $d=o$, ou seja, a série é estacionária não necessitando de transformações. O melhor modelo encontrado foi um AR(2), o critério de validação usado o MAPE que resultou 4,62\%, e o MSE igual a 1,5.

Através da figura 5 e 6 observa-se que a autocorrelação foi removida dos dados.

Figura 5 - Função de autocorrelação para os dados modelados

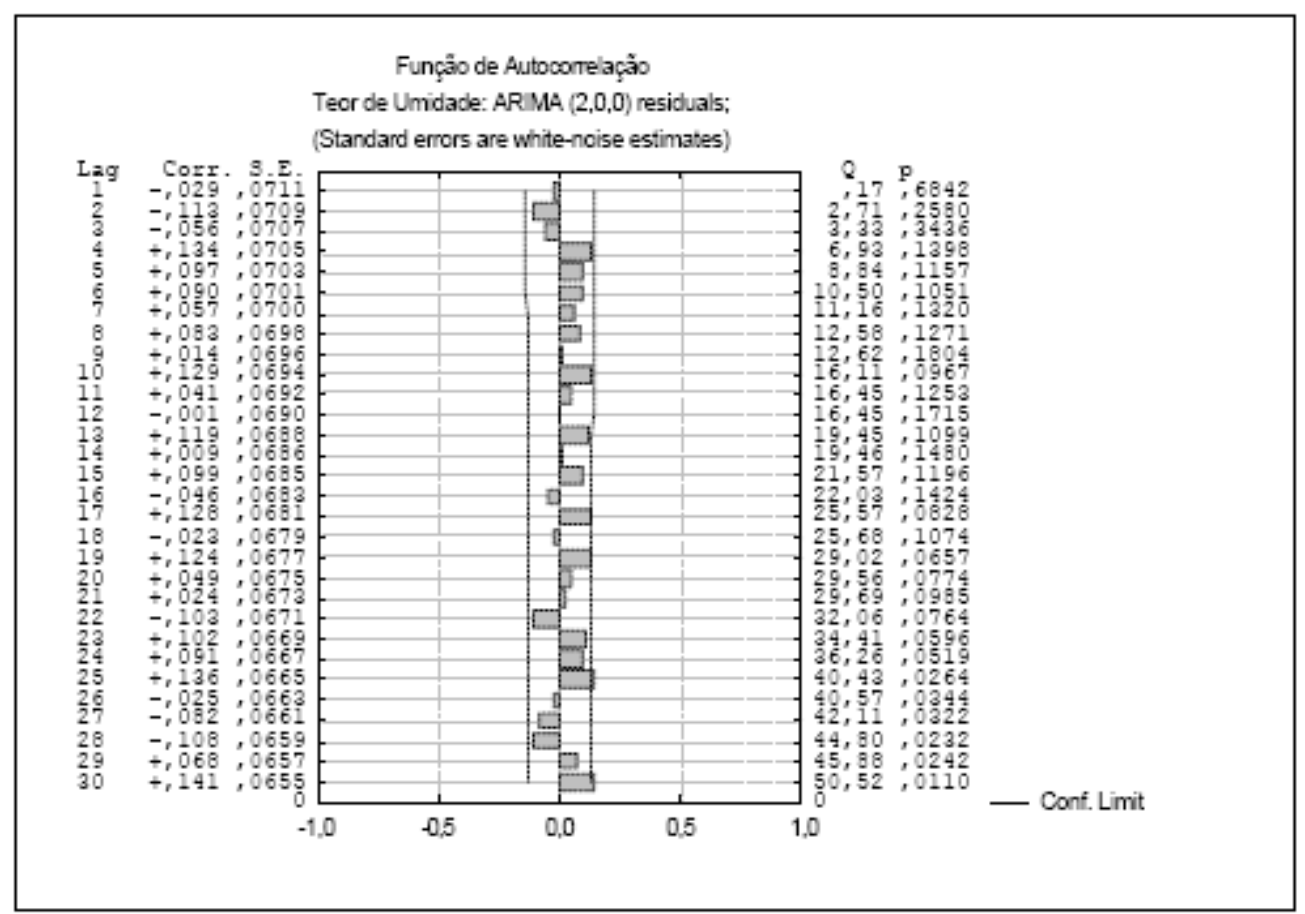

Figura 6 - Função de autocorrelação parcial para os dados modelados

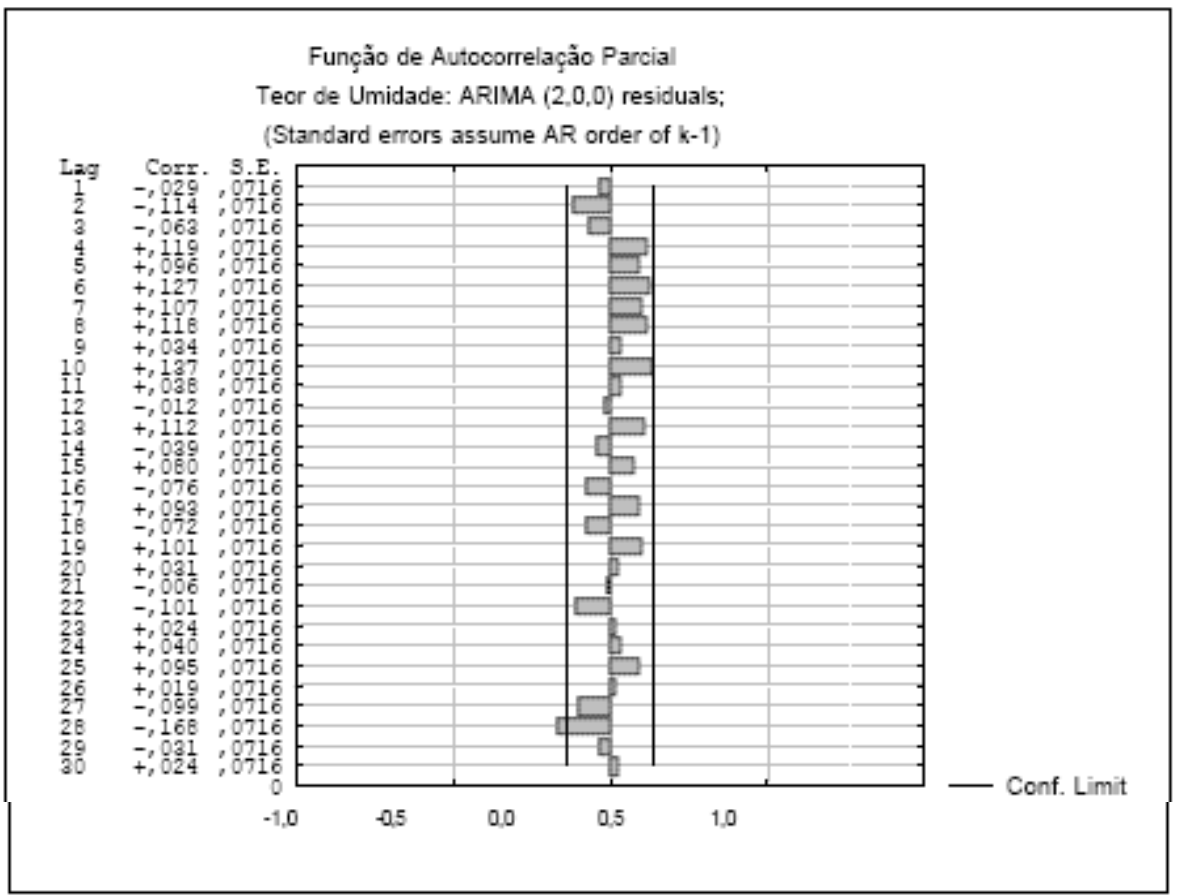


Na tabela 3 encontra-se o sumário dos parâmetros do modelo.

Tabela 3 - Sumário dos parâmetros do modelo

\begin{tabular}{lll}
\hline Critérios & Estimativa & Erro padrão \\
\hline Constante & 4,38 & 0,025 \\
& & 0,069 \\
$\mathrm{P}(1)$ & 0,37 & 0,069 \\
\hline
\end{tabular}

\section{Previsão}

Encontra-se a previsão através da equação:

$$
Z_{t+1}^{*}=\emptyset_{1} Z_{t}+\emptyset_{2} Z_{t-1}+\alpha_{t+1}
$$

onde $\mathrm{Z}_{\mathrm{t}+1}^{*}$ é o valor predito para o modelo no tempo $t+1$. A componente aleatória é obtida considerando o desvio padrão dos resíduos.

A tabela 4 mostra os valores observados e os previstos encontrados, e a figura 7 mostra o gráfico da previsão.

A tabela 4 mostra os valores observados e os previstos encontrados, e a figura 7 mostra o gráfico da previsão.

Tabela 4 - Valores observados e preditos

\begin{tabular}{ccc}
\hline Período & Observados & Preditos \\
\hline 25/outubro & 4,5 & 4,2719 \\
26/outubro & 4,4 & 4,2362 \\
27/outubro & 4,7 & 4,2973 \\
28/outubro & 4,5 & 4,3103 \\
29/outubro & 4,4 & 4,3318 \\
\hline
\end{tabular}




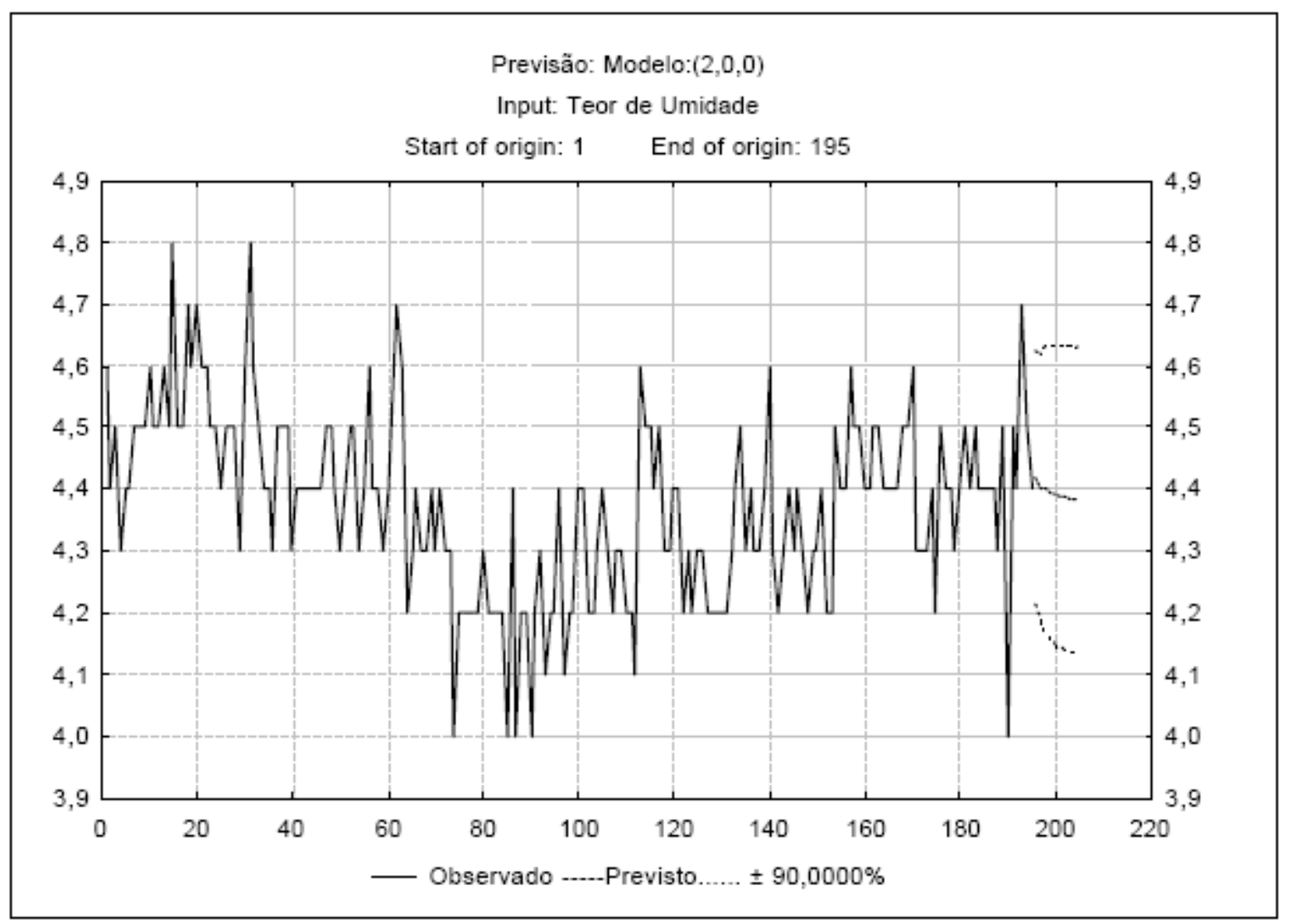

\title{
6. Conclusão e recomendações
}

Ao término do estudo relacionado obtiveram-se gráficos representativos, tabelas e demais cálculos estatísticos a fim de facilitar a modelagem via Box e Jenkins. Verificou-se que a série é estacionária $(d=0)$ e, encontrou-se uma equação representativa do teor de umidade da areia, o modelo autorregressivo $\operatorname{AR}(2)$, que permite simulações de previsões futuras da série, onde mais tarde, dando continuidade ao projeto, auxiliará na aplicação de um estudo via Gráfícos de Controle.

\begin{abstract}
In this study are verified the occurred alterations in the molds of casting produced for the industry FUNDIMISA. For this, it is necessary to find a model that represents the reality and that makes forecasts. Until the beginning of the years 70, studies involving time series tried to decompose them its components, after this date an alternative methodology was created, the Box and Jenkins methodology, where it demonstrates that the times series are generated integrally by a random mechanism. For the ARIMA(p,d,q) model, the number of successive differentiations to make that the original series $\left(Z_{t}\right)$ it becomes stationary. Then, the order of the function is determined and the parameters are estimated by the ARMA model. Together with responsible persons for the productive process, it was made the determination of the variables referred the elements involved in the assays of sand for foundry, that are: humidity text, permeability, resistance the compression, compactabilidade and plasticity of the sand, collected in march/2004 up to october/2004. The used data are representative of the analyzed daily number of the text of humidity of the sand, measured in $\%$. Note that the series has great variability, and the best model found was the AR(2), the criterion used was the MAPE with $0,52 \%$, and the MSE with 1,5 .
\end{abstract}


Key-words: Box and Jenkins, Time Series, Humidity

* This paper is supported by CAPES and FAPERGS

\section{Referências}

BELTRÃO. K. I. Séries temporais no domínio da freqüência: uma introdução. In: $4^{\mathrm{a}}$ Escola de Séries Temporais e Econometria. UFRJ/RJ. 1991.

BOX, G. E. P; JENKINS, G. M.. Times series analysis: forecasting and control. Holden-Day. San Francisco, 1976.

BOX, G. E. P ; JENKINS, G. M.; REINSEL, G. C. Times series analysis: forecasting and control, $3^{\mathrm{a}}$ Ed. San Francisco: Holden-Day, 1994.

ONG, C-S; H, J-J; T, G-H. Model identification of ARIMA family using genetic algorithms. Applied Mathematics and Computation. v.164 . pp. 885-912. 2005

cross ${ }^{\text {ref }}$

WEI; W. W. S. Time series analysis. Addison-Wesley Publishing Company. Inc. New York. P. 478. 1990.

ZHANG, G. P. Time series forecasting using a hybrid ARIMA and neural network model. Neurocomputing. 50.159-175. 2003.

\section{Dados completos do primeiro autor:}

Nome completo: Suzana Russo

Filiação institucional: Universidade Regional Integrada do Alto Uruguai e

das Missões - URI

Função ou cargo ocupado: Professora Tempo Integral

Endereço completo para correspondência (bairro, cidade, estado, país e CEP): Travessa Germano Tiefensee 239, Bairro Guelher, Santo Ângelo - RS.

Brasi, CEP: $98805-470$

Telefones para contato: 55-33132501; 55-99722711

e-mail: jss@urisan.tche.br

Recebido para publicação em: 04/12/2005

Aceito para publicação em: 11/02/2006 